\title{
OPERATOR VALUED BMO AND COMMUTATORS
}

\author{
Oscar Blasco
}

\begin{abstract}
If $E$ is a Banach space, $b \in B M O\left(\mathbb{R}^{n}, \mathcal{L}(E)\right)$ and $T$ is a $\mathcal{L}(E)$-valued Calderón-Zygmund type operator with operator-valued kernel $k$, we show the boundedness of the commutator $T_{b}(f)=$ $b T(f)-T(b f)$ on $L^{p}\left(\mathbb{R}^{n}, E\right)$ for $1<p<\infty$ whenever $b$ and $k$ verify some commuting properties. Some endpoint estimates are also provided.
\end{abstract}

\section{Introduction and notation}

We shall work on $\mathbb{R}^{n}$ endowed with the Lebesgue measure $d x$ and use the notation $|A|=\int_{A} d x$. Given a Banach space $(X,\|\cdot\|)$ and $1 \leq$ $p<\infty$ we shall denote by $L^{p}\left(\mathbb{R}^{n}, X\right)$ the space of Bochner $p$-integrable functions endowed with the norm $\|f\|_{L^{p}\left(\mathbb{R}^{n}, X\right)}=\left(\int_{\mathbb{R}^{n}}\|f(x)\|^{p} d x\right)^{1 / p}$, by $L_{c}^{\infty}\left(\mathbb{R}^{n}, X\right)$ the closure of the compactly supported functions in $L^{\infty}\left(\mathbb{R}^{n}, X\right)$ and by $L_{\text {weak }, \alpha}\left(\mathbb{R}^{n}, X\right)$ the space of measurable functions such that $\left|\left\{x \in \mathbb{R}^{n}:\|f(x)\|>\lambda\right\}\right| \leq \alpha(\lambda)$ where $\alpha: \mathbb{R}^{+} \rightarrow \mathbb{R}^{+}$is a non increasing function. We write $H^{1}\left(\mathbb{R}^{n}, X\right)$ for the Hardy space defined by $X$-valued atoms, that is the space of integrable functions $f=\sum_{k} \lambda_{k} a_{k}$ where $\lambda_{k} \in \mathbb{R}, \sum_{k}\left|\lambda_{k}\right|<\infty$ and $a_{k}$ belong to $L_{c}^{\infty}\left(\mathbb{R}^{n}, X\right), \operatorname{supp}\left(a_{k}\right) \subset$ $Q_{k}$ for some cube $Q_{k}, \int_{Q_{k}} a(x) d x=0$ and $\|a(x)\| \leq \frac{1}{\mid Q_{k}}$. We also write, for a positive function $\phi$ defined on $\mathbb{R}^{+}, B M O_{\phi}\left(\mathbb{R}^{n}, X\right)$ for the space of locally integrable functions such that there exists $C>0$ such that for all cube $Q$

$$
\frac{1}{|Q|} \int_{Q}\left\|f(x)-f_{Q}\right\| d x \leq C \phi(|Q|)
$$

2000 Mathematics Subject Classification. Primary: 42B20; Secondary: 42B25.

Key words. Bounded mean oscillation, commutator, operator-valued singular integrals.

Partially supported by Proyecto MTM 2005-08350. 
where $f_{Q}=\frac{1}{|Q|} \int_{Q} f(x) d x$. For $\phi(t)=1$ we denote the space $B M O\left(\mathbb{R}^{n}, X\right)$ and the above condition is equivalent to

$$
\operatorname{osc}_{p}(f, Q)=\left(\frac{1}{|Q|} \int_{Q}\left\|f(x)-f_{Q}\right\|^{p} d x\right)^{1 / p}<\infty
$$

for each (equivalently for all) $1 \leq p<\infty$. The infimum of the constants satisfying the above inequalities define the "norm" in the space.

Let us denote by $f^{\#}$ and $M(f)$ the sharp and the Hardy-Littlewood maximal functions of $f$ defined by

$$
f^{\#}(x)=\sup _{x \in Q} \operatorname{osc}_{1}(f, Q) \quad \text { and } \quad M(f)(x)=\sup _{x \in Q} \frac{1}{|Q|} \int_{Q}\|f(x)\| d x .
$$

We write $M_{q}(f)=M\left(\|f\|^{q}\right)^{1 / q}$ for $1 \leq q<\infty$.

It is well known that

$$
f^{\#}(x) \approx \sup _{x \in Q} \inf _{c_{Q} \in X} \frac{1}{|Q|} \int_{Q}\left\|f(x)-c_{Q}\right\| d x
$$

and that $f^{\#} \in L^{p}\left(\mathbb{R}^{n}\right)$ implies that $f \in L^{p}\left(\mathbb{R}^{n}, X\right)$ for $1<p<\infty$.

Recall also that $M_{q}$ maps $L^{q}\left(\mathbb{R}^{n}, X\right)$ into $L_{\text {weak }, 1 / t^{q}}$ and

$$
M_{q}: L^{p}\left(\mathbb{R}^{n}, X\right) \rightarrow L^{p}\left(\mathbb{R}^{n}\right) \text { is bounded for } q<p \leq \infty .
$$

Throughout the paper $E$ denotes a Banach space and $\mathcal{L}(E)$ denotes the space of bounded linear operators on $E$.

Definition 1.1. We shall say that $T$ is a $\mathcal{L}(E)$-Calderón-Zygmund type operator if the following properties are fulfilled:

(3) $T: L^{p}\left(\mathbb{R}^{n}, E\right) \rightarrow L^{p}\left(\mathbb{R}^{n}, E\right)$ is bounded for some $1<p<\infty$,

there exists a locally integrable function $k$ from $\mathbb{R}^{n} \times \mathbb{R}^{n} \backslash\{(x, x)\}$ into $\mathcal{L}(E)$ such that

$$
T f(x)=\int k(x, y) f(y) d y
$$

for every $E$-valued bounded and compactly supported function $f$ and $x \notin \operatorname{supp} f$, and there exists $\varepsilon>0$ such that

$$
\left\|k(x, y)-k\left(x^{\prime}, y\right)\right\| \leq C \frac{\left|x-x^{\prime}\right|^{\varepsilon}}{|x-y|^{n+\varepsilon}}, \quad|x-y| \geq 2\left|x-x^{\prime}\right| .
$$


Remark 1.2. It is well known (see $[\mathbf{R R T}]$ or $[\mathbf{G R}]$ ) that in such a case $T$ is bounded on $L^{q}\left(\mathbb{R}^{n}, E\right)$ for any $1<q<\infty$.

Throughout the literature, after the result on commutators in $[\mathbf{C R W}]$, many results appeared in connection with the boundedness of commutators of Calderón-Zygmund type operators and multiplication by a function $b$ given by $T_{b}(f)=b T(f)-T(b f)$ on many different function spaces and on their weighted and vector-valued versions (see [ST1], [ST2], [ST3], [ST4], [ST5]). Also endpoint estimates for the commutator was a topic that attracted several people on different directions (see $[\mathbf{C P}]$, $[\mathbf{H S T}],[\mathbf{P P}],[\mathbf{P 1}],[\mathbf{P 2}],[\mathbf{P T 1}],[\mathbf{P T 2}])$.

We shall deal in this paper with the unweighted but operator-valued version of the commutators and will give some results about its boundedness on $L^{p}\left(\mathbb{R}^{n}, E\right)$ and produce some endpoint estimates.

The following result was shown by C. Segovia and J. L. Torrea (even with some weights and two different Banach spaces).

Theorem 1.3 ([ST1, Theorem 1]). Let $T$ be an $\mathcal{L}(E)$-valued CalderónZygmund type operator and let $\ell \rightarrow \bar{\ell}$ be a correspondence from $\mathcal{L}(E)$ to $\mathcal{L}(E)$ such that

$$
\tilde{\ell} T(f)(x)=T(\ell f)(x)
$$

and

$$
k(x, y) \ell=\tilde{\ell} k(x, y)
$$

If $b$ is an $\mathcal{L}(E)$-valued function such that $b$ and $\tilde{b}$ belong to $B M O\left(\mathbb{R}^{n}, \mathcal{L}(E)\right)$ then

$$
T_{b}(f)=b T(f)-T(b f)
$$

is bounded from $L^{p}\left(\mathbb{R}^{n}, E\right) \rightarrow L^{p}\left(\mathbb{R}^{n}, E\right)$ for all $1<p<\infty$.

The endpoint estimates of that result were later studied by E. Harboure, C. Segovia and J. L. Torrea (see Theorem A and Theorem 3.1 in $[$ HST] $)$ when $b$ was assumed to be scalar-valued. From their results one concludes that non-constant scalar valued $B M O$ functions do not define bounded commutators from $L^{\infty}\left(\mathbb{R}^{n}, E\right)$ to $B M O\left(\mathbb{R}^{n}, F\right)$ when the kernel of the Calderón-Zygmund type operators are $\mathcal{L}(E, F)$-valued. Also it was shown that, in general, $T_{b}$ does not map $H^{1}\left(\mathbb{R}^{n}, E\right)$ into $L^{1}(\mathbb{R}, F)$. 
The aim of this note is to use the techniques developed in the papers $[\mathbf{S T 1}],[\mathbf{H S T}]$ to get some extensions for operator-valued BMOfunctions having some commuting properties with the kernel. In particular we show that if $\|k(x, y)\| \leq \psi\left(|x-y|^{n}\right)$ for certain function $\psi$ then the commutators of operator-valued $B M O$ functions and operator-valued Calderón-Zygmund operators map $L_{c}^{\infty}\left(\mathbb{R}^{n}, E\right)$ into $B M O_{\phi}\left(\mathbb{R}^{n}, E\right)$ for a function $\phi$ depending on $\psi$. Also we shall see that the commutator is bounded from $H^{1}\left(\mathbb{R}^{n}, E\right)$ into $L_{\text {weak, } \alpha}\left(\mathbb{R}^{n}, E\right)$ for a suitable $\alpha$ defined from $\psi$.

Throughout the paper $b: \mathbb{R}^{n} \rightarrow \mathcal{L}(E)$ is locally integrable and $T$ is a Calderón-Zygmund type operator defined on $L^{p}\left(\mathbb{R}^{n}, E\right)$ with a kernel $k$ satisfying (3), (4) and (5). We write

$$
T_{b}(f)(x)=b(x)(T(f)(x))-T(b f)(x)
$$

where we understand the product $b f$ as the $E$-valued function $b(y)(f(y))$.

We shall use the notation $Q$ for a cube in $\mathbb{R}^{n}, x_{Q}$ for its center, $\ell(Q)$ for the side length, $\lambda Q$ for a cube centered at $x_{Q}$ with side length $\lambda \ell(Q)$ and $Q^{c}=\mathbb{R}^{n} \backslash Q$. Finally, as usual, $C$ stands for a constant that may vary from line to line.

\section{The results}

We improve Theorem 1.3 by realizing that conditions (6) and (7) are not of independent nature. Our basic assumptions throughout the paper are the following ones:

$$
b(z) k(x, y)=k(x, y) b(z), \quad x, y, z \in \mathbb{R}^{n}, x \neq y .
$$

(A2) $b_{Q} T\left(e \chi_{A}\right)(x)=T\left(b_{Q} e \chi_{A}\right)(x), \quad x \in Q, A \subseteq Q$ measurable, $e \in E$.

We would like to point out that (A1) produces the following cancelation property.

Lemma 2.1. Let $b$ satisfy (A1), let $Q, Q^{\prime}$ be cubes in $\mathbb{R}^{n}$ and $f_{1}$ and $f_{2}$ be compactly supported E-valued with supp $f_{1} \subset Q^{\prime}$ and $\operatorname{supp} f_{2} \subset$ $\left(Q^{\prime}\right)^{c}$. Then

$$
\begin{array}{ll}
b_{Q} T\left(f_{2}\right)(x)=T\left(b_{Q} f_{2}\right)(x), & x \in Q^{\prime} . \\
b_{Q} T\left(f_{1}\right)(x)=T\left(b_{Q} f_{1}\right)(x), & x \in\left(Q^{\prime}\right)^{c} .
\end{array}
$$


Proof: Let us show (8). Recall that if $F \in L^{1}\left(\mathbb{R}^{n}, X\right)$ and $\Phi \in \mathcal{L}(X)$ for a given Banach space $X$ then $\Phi\left(\int F(x) d x\right)=\int \Phi F(x) d x$. Hence, considering $X=\mathcal{L}(E)$ and $\Phi(T)=T b_{Q}$ or $\Phi(T)=b_{Q} T$ one gets, for $x \in Q^{\prime}$,

$$
\begin{aligned}
b_{Q} T\left(f_{2}\right)(x) & =b_{Q}\left(\int_{\left(Q^{\prime}\right)^{c}} k(x, y) f_{2}(y) d y\right) \\
& =\int_{\left(Q^{\prime}\right)^{c}} b_{Q} k(x, y) f_{2}(y) d y \\
& =\int_{\left(Q^{\prime}\right)^{c}}\left(\frac{1}{|Q|} \int_{Q} b(z) d z\right) k(x, y) f_{2}(y) d y \\
& =\int_{\left(Q^{\prime}\right)^{c}}\left(\frac{1}{|Q|} \int_{Q} b(z) k(x, y) d z\right) f_{2}(y) d y \\
& =\int_{\left(Q^{\prime}\right)^{c}} k(x, y)\left(\frac{1}{|Q|} \int_{Q} b(z) d z\right) f_{2}(y) d y \\
& =T\left(b_{Q} f_{2}\right)(x) .
\end{aligned}
$$

(9) follows similarly and it is left to the reader.

The assumptions (A1) and (A2) hold true, for instance, in the following cases.

Example 2.2. Let $T, S$ be operators in $\mathcal{L}(E)$ with $S T=T S$. Let $b(x)=b_{0}(x) T$ and $k(x, y)=k_{0}(x, y) S$ for scalar valued functions $b_{0}$ and $k_{0}$.

Hence our results will apply whenever either $b$ or $k$ are scalar-valued.

Example 2.3. Let $E$ be a Banach space, $b_{0}(x) \in E^{*}$ and let $k(x, y)$ be scalar valued function such that $T$ is bounded on $L^{p}\left(\mathbb{R}^{n}, E\right)$. The case $T_{b_{0}}(f)=\left\langle b_{0}, T(f)\right\rangle-T\left(\left\langle b_{0}, f\right\rangle\right)$ follows from the operator-valued case by selecting $e \in E$ and $b(x)(f)=\left\langle b_{0}(x), f\right\rangle e$ in $\mathcal{L}(E)$.

We formulate now the results of the paper. The first one is just a modification of a similar result from [ST1] but stated here under slightly weaker assumptions. 
Theorem 2.4. Let $b \in B M O\left(\mathbb{R}^{n}, \mathcal{L}(E)\right)$ and let $T$ be a Calderón-Zygmund type operator defined on $L^{p}\left(\mathbb{R}^{n}, E\right)$ where the kernel and $b$ satisfy (A1) and (A2). Then $T_{b}$ is bounded on $L^{p}\left(\mathbb{R}^{n}, E\right)$ for any $1<p<$ $\infty$.

Next we analyze the endpoint estimates. We construct a function $\phi$ for the commutator $T_{b}$ to be bounded from $L_{c}^{\infty}\left(\mathbb{R}^{n}, E\right)$ into $B M O_{\phi}\left(\mathbb{R}^{n}, \mathcal{L}(E)\right)$.

Theorem 2.5. Let $T$ be a Calderón-Zygmund type operator with operator-valued kernel $k$ and assume that

$$
\|k(x, y)\| \leq \psi\left(|x-y|^{n}\right), \quad x \neq y
$$

for some $\psi: \mathbb{R}^{+} \rightarrow \mathbb{R}^{+}$such that $\int_{s}^{\infty} \psi(u) d u=\phi(s)<\infty$ for all $s>0$.

If $b \in B M O\left(\mathbb{R}^{n}, \mathcal{L}(E)\right)$ satisfies $(\mathbf{A} \mathbf{1})$ and that $T_{b}$ is bounded on some $L^{p}\left(\mathbb{R}^{n}, E\right)$ then $T_{b}$ is bounded from $L_{c}^{\infty}\left(\mathbb{R}^{n}, E\right)$ into $B M O_{1+\phi}\left(\mathbb{R}^{n}, E\right)$.

We also find a function $\alpha$ such that the commutator of a function $b$ in $B M O\left(\mathbb{R}^{n}, \mathcal{L}(E)\right)$ with a Calderón-Zygmund type operator $T_{b}$ maps the space $H^{1}\left(\mathbb{R}^{n}, E\right)$ into $L_{\text {weak }, \alpha}\left(\mathbb{R}^{6} n, E\right)$.

Theorem 2.6. Let $T$ be a Calderón-Zygmund type operator with operator-valued kernel $k$. Assume that

$$
\|k(x, y)\| \leq \gamma\left(|x-y|^{n}\right), \quad x \neq y
$$

for some decreasing function $\gamma: \mathbb{R}^{+} \rightarrow \mathbb{R}^{+}$and

$$
\left\|k(x, y)-k\left(x, y^{\prime}\right)\right\| \leq C \frac{\left|y-y^{\prime}\right|^{\varepsilon}}{|x-y|^{n+\varepsilon}}, \quad|x-y| \geq 2\left|y-y^{\prime}\right| .
$$

If $b \in B M O\left(\mathbb{R}^{n}, \mathcal{L}(E)\right)$ satisfies ( $\left.\mathbf{A 1}\right)$ and $T_{b}$ is bounded on some $L^{p}\left(\mathbb{R}^{n}, E\right)$ then $T_{b}$ is bounded from $H^{1}\left(\mathbb{R}^{n}, E\right)$ into $L_{\text {weak }, \alpha}^{1}\left(\mathbb{R}^{n}, E\right)$ for $\alpha(\lambda)=\gamma^{-1}\left(\|b\|_{B M O}^{-1} \lambda\right)$.

As corollaries of these results one obtains the following applications.

Corollary 2.7 (see [ST1]). Let $H$ be the Hilbert transform

$$
H(f)(x)=p \cdot v \cdot \int \frac{f(y)}{x-y} d y,
$$

and $E$ be a $U M D$ space (see $[\mathbf{G R}])$. If $b \in B M O(\mathbb{R}, \mathcal{L}(E))$ then

(i) $H_{b}$ maps $L^{p}(\mathbb{R}, E)$ to $L^{p}(\mathbb{R}, E)$ for $1<p<\infty$ and

(ii) $H_{b}$ maps $H^{1}(\mathbb{R}, E)$ to $L_{\text {weak }, 1 / t}(\mathbb{R}, E)$.

Although our results are stated in $\mathbb{R}$, similar ones work in $\mathbb{T}$. In this case we can obtain 
Corollary 2.8 (see [HST]). Let $\tilde{H}$ be the conjugate function in the torus

$$
\tilde{H}(f)(x)=p \cdot v \cdot \frac{1}{2 \pi} \int \cot \left(\frac{x-y}{2}\right) f(y) d y, \quad x \in[-\pi, \pi]
$$

and $E$ be a $U M D$ space. If $b \in B M O(\mathbb{R}, \mathcal{L}(E))$ then

(i) $H_{b}$ maps $L^{p}(\mathbb{T}, E)$ to $L^{p}(\mathbb{T}, E)$ for $1<p<\infty$,

(ii) $H_{b}$ maps $H^{1}(\mathbb{T}, E)$ to $L_{\text {weak }, 1 / t}(\mathbb{T}, E)$ and

(iii) $H_{b}$ maps $L^{\infty}(\mathbb{T}, E)$ to $B M O_{|\log t|^{-1}}(\mathbb{T}, E)$.

\section{Proof of the results}

Let us start by showing some consequences from (A1) and (A2).

Lemma 3.1. Let b satisfy (A1) and (A2), $Q$ be a cube in $\mathbb{R}^{n}$ and $f$ be simple E-valued function. Then

$$
b_{Q} T(f)(x)=T\left(b_{Q} f\right)(x), \quad x \in Q
$$

Proof: Take $f_{1}=f \chi_{Q}$ and $f_{2}=f-f_{1}$. Using Lemma 2.1 one obtains $b_{Q} T\left(f_{2}\right) \chi_{Q}=T\left(b_{Q} f_{2}\right) \chi_{Q}$ and (A2) shows that $b_{Q} T\left(f_{1}\right) \chi_{Q}=$ $T\left(b_{Q} f_{1}\right) \chi_{Q}$.

The following useful lemma is essentially included in [HST].

Lemma 3.2. Let $Q$ be a cube, denote $Q_{j}=2^{j} Q$ and let $f$ be compactly supported E-valued with supp $f \subset(2 Q)^{c}$. Then there exists $C>0$ such that

(14) $\left\|T(f)(x)-T(f)\left(x^{\prime}\right)\right\| \leq C \frac{\left|x-x^{\prime}\right|^{\varepsilon}}{\ell(Q)^{\varepsilon}} \sum_{j=2}^{\infty} \frac{2^{-j \varepsilon}}{\left|Q_{j}\right|} \int_{Q_{j}}\|f(y)\| d y, \quad x, x^{\prime} \in Q$. 
Proof: Using (4) and (5) one has

$$
\begin{aligned}
\left\|T(f)(x)-T(f)\left(x^{\prime}\right)\right\| & \leq \int_{(2 Q)^{c}}\left\|k(x, y)-k\left(x^{\prime}, y\right)\right\|\|f(y)\| d y \\
& \leq C\left|x-x^{\prime}\right|^{\varepsilon} \int_{(2 Q)^{c}} \frac{\|f(y)\|}{|x-y|^{n+\varepsilon}} d y \\
& \leq C\left|x-x^{\prime}\right|^{\varepsilon} \sum_{j=1}^{\infty} \int_{Q_{j+1}-Q_{j}} \frac{\|f(y)\|}{|x-y|^{n+\varepsilon}} d y \\
& \leq C\left|x-x^{\prime}\right|^{\varepsilon} \sum_{j=2}^{\infty} \frac{1}{\ell\left(Q_{j}\right)^{n+\varepsilon}} \int_{Q_{j}}\|f(y)\| d y \\
& \leq C \frac{\left|x-x^{\prime}\right|^{\varepsilon}}{\ell(Q)^{\varepsilon}} \sum_{j=2}^{\infty} 2^{-j \varepsilon} \frac{1}{\left|Q_{j}\right|} \int_{Q_{j}}\|f(y)\| d y .
\end{aligned}
$$

Proof of Theorem 2.4: Let $f$ be a simple $E$-valued function. Let $Q$ be a cube, $f_{1}=f \chi_{2 Q}$ and $f_{2}=f-f_{1}$. Put $c_{Q}=T\left(\left(b_{Q}-b\right) f_{2}\right)\left(x_{Q}\right)$.

For each $x \in Q$ one has, applying Lemma 3.1,

$$
T_{b} f(x)-c_{Q}=\sum_{i=1}^{3} \sigma_{i}(x)
$$

where

$$
\begin{aligned}
& \sigma_{1}(x)=\left(b-b_{Q}\right) T f(x), \\
& \sigma_{2}(x)=T\left(\left(b_{Q}-b\right) f_{1}\right)(x)
\end{aligned}
$$

and

$$
\sigma_{3}(x)=T\left(\left(b_{Q}-b\right) f_{2}\right)(x)-T\left(\left(b_{Q}-b\right) f_{2}\right)\left(x_{Q}\right) .
$$

Observe that for $1<q<\infty$ and $1 / q+1 / q^{\prime}=1$ we can write

$$
\frac{1}{|Q|} \int_{Q}\left\|\sigma_{1}(x)\right\| d x \leq \operatorname{osc}_{q^{\prime}}(b, Q)\left(\frac{1}{|Q|} \int_{Q}\|T f(x)\|^{q} d x\right)^{1 / q} \text {. }
$$


For any $q>q_{1}>1$ one can use Remark 1.2 , for $1 / r+1 / q=1 / q_{1}$, to obtain

$$
\begin{aligned}
& \frac{1}{|Q|} \int_{Q}\left\|\sigma_{2}(x)\right\| d x \leq\left(\frac{1}{|Q|} \int_{Q}\left\|T\left(b_{Q}-b\right) f_{1}(x)\right\|^{q_{1}} d x\right)^{1 / q_{1}} \\
& \leq C\|T\|_{\mathcal{L}\left(L^{q_{1}}\left(\mathbb{R}^{n}, E\right)\right)}\left(\frac{1}{|Q|} \int_{Q}\left\|\left(b-b_{Q}\right) f_{1}(x)\right\|^{q_{1}} d x\right)^{1 / q_{1}} \\
& \leq C\|T\|_{\mathcal{L}\left(L^{q_{1}}\left(\mathbb{R}^{n}, E\right)\right)} \operatorname{osc}_{r}(b . Q)\left(\frac{1}{|Q|} \int_{Q}\|f(x)\|^{q} d x\right)^{1 / q} .
\end{aligned}
$$

Using Lemma 3.2, and taking into account that $\left\|b_{Q}-b_{2 Q}\right\| \leq C \operatorname{osc}_{q_{1}}(b, 2 Q)$, we also can estimate

$$
\begin{aligned}
\left\|\sigma_{3}(x)\right\| & \leq C \sum_{j=2}^{\infty} 2^{-j \varepsilon} \frac{1}{\left|Q_{j}\right|} \int_{Q_{j}}\left\|\left(b(y)-b_{Q}\right) f(y)\right\| d y \\
& \leq C \sum_{j=2}^{\infty} 2^{-j \varepsilon}\left(\frac{1}{\left|Q_{j}\right|} \int_{Q_{j}}\left\|b(y)-b_{Q}\right\|^{q^{\prime}} d y\right)^{1 / q^{\prime}}\left(\frac{1}{\left|Q_{j}\right|} \int_{Q_{j}}\|f(y)\|^{q} d y\right)^{1 / q} \\
& \leq C \sum_{j=2}^{\infty} 2^{-j \varepsilon}\left(\sum_{k=2}^{j} \operatorname{osc}_{q^{\prime}}\left(b, Q_{k}\right)\right)\left(\frac{1}{\left|Q_{j}\right|} \int_{Q_{j}}\|f(y)\|^{q} d y\right)^{1 / q} \\
& \leq C \sup _{j \geq 2}\left(\frac{1}{\left|Q_{j}\right|} \int_{Q_{j}}\|f(y)\|^{q} d y\right)^{1 / q}\left(\sum_{j=2}^{\infty} 2^{-j \varepsilon}\left(\sum_{k=2}^{j} \operatorname{osc}_{q^{\prime}}\left(b, Q_{k}\right)\right)\right) \\
& \leq C\|b\|_{B M O} \sup _{j \geq 2}\left(\frac{1}{\left|Q_{j}\right|} \int_{Q_{j}}\|f(y)\|^{q} d y\right)^{1 / q} \sum_{j} j 2^{-j \varepsilon} .
\end{aligned}
$$

Hence, combining the previous estimates, one obtains

$$
T_{b}(f)^{\#}(x) \leq C\|b\|_{B M O}\left(M_{q}(T f)(x)+M_{q}(f)(x)\right) .
$$

Now, for a given $1<p<\infty$, select $1<q<p$ and apply (2), which, combined with the boundedness of $T$ on $L^{p}\left(\mathbb{R}^{n}, E\right)$, shows that $\left\|T_{b}(f)^{\#}\right\|_{L^{p}\left(\mathbb{R}^{n}\right)} \leq C\|f\|_{L^{p}\left(\mathbb{R}^{n}, E\right)}$. Now use the vector-valued analogue of Fefferman-Stein's result (see $[\mathbf{F S}],[\mathbf{R R T}]$ ) to obtain that $\left\|T_{b}(f)\right\|_{L^{p}\left(\mathbb{R}^{n}, E\right)} \leq C\|f\|_{L^{p}\left(\mathbb{R}^{n}, E\right)}$. 
Proof of Theorem 2.5: As in the previous theorem, let $f$ be a simple $E$-valued function. Let $Q$ be a cube, $f_{1}=f \chi_{2 Q}, f_{2}=f-f_{1}$ and $c_{Q}=T\left(\left(b_{Q}-b\right) f_{2}\right)\left(x_{Q}\right)$ Now, using Lemma 2.1, we write

$$
T_{b} f(x)=T_{b}\left(f_{1}\right)(x)+\left(b(x)-b_{Q}\right) T\left(f_{2}\right)(x)+T\left(\left(b_{Q}-b\right) f_{2}\right)(x) .
$$

Denote now

$$
\begin{aligned}
& \sigma_{1}(x)=T_{b}\left(f_{1}\right)(x), \\
& \sigma_{2}(x)=\left(b(x)-b_{Q}\right) T\left(f_{2}\right)(x), \\
& \sigma_{3}(x)=T\left(\left(b_{Q}-b\right) f_{2}\right)(x)-T\left(\left(b_{Q}-b\right) f_{2}\right)\left(x_{Q}\right) .
\end{aligned}
$$

Hence $T_{b} f-c_{Q}=\sum_{i=1}^{3} \sigma_{i}$. Note that the boundedness of $T_{b}$ on $L^{p}\left(\mathbb{R}^{n}, E\right)$ gives

$$
\frac{1}{|Q|} \int_{Q}\left\|\sigma_{1}(x)\right\| d x \leq C\left\|T_{b}\right\|_{\mathcal{L}\left(L^{p}\right)}\left(\frac{1}{|2 Q|} \int_{2 Q}\|f(x)\|^{p} d x\right)^{1 / p} \leq C\|f\|_{\infty} .
$$

On the other hand

$$
\begin{aligned}
\frac{1}{|Q|} \int_{Q}\left\|\sigma_{2}(x)\right\| d x & \leq \frac{1}{|Q|} \int_{Q}\left\|b(x)-b_{Q}\right\|\left\|\int_{(2 Q)^{c}} k(x, y) f(y) d y\right\| d x \\
& \leq C \frac{1}{|Q|} \int_{Q}\left\|b(x)-b_{Q}\right\|\left(\int_{(2 Q)^{c}} \psi\left(|x-y|^{n}\right)\|f(y)\| d y\right) d x \\
& \leq C\|f\|_{\infty} \frac{1}{|Q|} \int_{Q}\left\|b(x)-b_{Q}\right\|\left(\int_{|u|>\ell(Q)} \psi\left(|u|^{n}\right) d u\right) d x \\
& \leq C\|f\|_{\infty}\left(\frac{1}{|Q|} \int_{Q}\left\|b(x)-b_{Q}\right\| d x\right)\left(\int_{\ell(Q)}^{\infty} r^{n-1} \psi\left(r^{n}\right) d r\right) \\
& \leq C\|f\|_{\infty}\|b\|_{B M O}\left(\int_{|Q|}^{\infty} \psi(t) d t\right) .
\end{aligned}
$$

Finally Lemma 3.2 gives immediately

$$
\frac{1}{|Q|} \int_{Q}\left\|\sigma_{3}(x)\right\| d x \leq C\|b\|_{B M O}\|f\|_{\infty}
$$

This allows us to conclude the estimate

$$
\frac{1}{|Q|} \int_{Q}\left\|T_{b} f(x)-c_{Q}\right\| d x \leq C\|f\|_{\infty}(1+\phi(|Q|)) .
$$

This shows that $T_{b}$ maps $L_{c}^{\infty}\left(\mathbb{R}^{n}, E\right)$ into $B M O_{1+\phi}\left(\mathbb{R}^{n}, E\right)$. 
Proof of Theorem 2.6: Let $a$ be an $E$-valued atom supported on $Q$. Using Lemma 2.1 again we can write

$$
\begin{aligned}
T_{b} a(x)=\chi_{2 Q}(x) T_{b}(a)(x)+\chi_{(2 Q)^{c}}(x)\left(b(x)-b_{Q}\right) T(a)(x) \\
+\chi_{(2 Q)^{c}}(x) T\left(\left(b_{Q}-b\right) a\right)(x) .
\end{aligned}
$$

Denote now

$$
\begin{aligned}
& \sigma_{1}(x)=\chi_{2 Q}(x) T_{b}(a)(x), \\
& \sigma_{2}(x)=\chi_{(2 Q)^{c}}(x)\left(b(x)-b_{Q}\right) T(a)(x), \\
& \sigma_{3}(x)=\chi_{(2 Q)^{c}}(x) T\left(\left(b_{Q}-b\right) a\right)(x) .
\end{aligned}
$$

Now, using the boundedness of $T_{b}$ on $L^{p}\left(\mathbb{R}^{n}, E\right)$,

$$
\begin{aligned}
\int_{\mathbb{R}^{n}}\left\|\sigma_{1}(x)\right\| d x & \leq C|Q|^{1 / p^{\prime}}\left\|T_{b}(a)\right\|_{L^{p}\left(\mathbb{R}^{n}, E\right)} \\
& \leq C\left\|T_{b}\right\|_{\mathcal{L}\left(L^{p}\right)}|Q|\left(\frac{1}{|Q|} \int_{Q}\|a(x)\|^{p} d x\right)^{1 / p} \\
& \leq C\left\|T_{b}\right\|_{\mathcal{L}\left(L^{p}\right)} .
\end{aligned}
$$

Also we have

$$
\begin{aligned}
\int_{\mathbb{R}^{n}}\left\|\sigma_{2}(x)\right\| d x & \leq \int_{(2 Q)^{c}}\left\|b(x)-b_{Q}\right\|\left\|\int_{Q} k(x, y) a(y) d y\right\| d x \\
& \leq \int_{(2 Q)^{c}}\left\|b(x)-b_{Q}\right\|\left\|\int_{Q}\left(k(x, y)-k\left(x, x_{Q}\right)\right) a(y) d y\right\| d x \\
& \leq C \int_{(2 Q)^{c}}\left\|b(x)-b_{Q}\right\|\left(\int_{Q} \frac{\left|y-x_{Q}\right|^{\varepsilon}}{|x-y|^{n+\varepsilon}}\|a(y)\| d y\right) d x \\
& \leq C \frac{\ell(Q)^{\varepsilon}}{|Q|} \int_{Q}\left(\int_{(2 Q)^{c}} \frac{\left\|b(x)-b_{Q}\right\|}{|x-y|^{n+\varepsilon}} d x\right) d y \\
& \leq C \frac{\ell(Q)^{\varepsilon}}{|Q|} \int_{Q}\left(\sum_{j=2}^{\infty} \frac{1}{\ell\left(Q_{j}\right)^{n+\varepsilon}} \int_{Q_{j}-Q_{j-1}}\left\|b(x)-b_{Q}\right\| d x\right) d y \\
& \leq C\left(\sum_{j=2}^{\infty} 2^{-j \varepsilon} \frac{1}{\left|Q_{j}\right|} \int_{Q_{j}}\left\|b(x)-b_{Q}\right\| d x\right) \leq C \|_{B M O}
\end{aligned}
$$


Now decompose $\sigma_{3}=\sigma_{3,1}+\sigma_{3,2}$ where

$$
\begin{aligned}
& \sigma_{3,1}(x)=\chi_{(2 Q)^{c}}(x) \int_{Q}\left(k(x, y)-k\left(x, x_{Q}\right)\right)\left(b_{Q}-b(y)\right) a(y) d y, \\
& \sigma_{3,2}(x)=\chi_{(2 Q)^{c}}(x) k\left(x, x_{Q}\right) \int_{Q} b(y) a(y) d y .
\end{aligned}
$$

Therefore

$$
\begin{aligned}
\int_{\mathbb{R}^{n}}\left\|\sigma_{3,1}(x)\right\| d x & \leq \int_{(2 Q)^{c}} \int_{Q}\left\|k(x, y)-k\left(x, x_{Q}\right)\right\|\left\|b_{Q}-b(y)\right\|\|a(y)\| d y d x \\
& \leq \int_{(2 Q)^{c}} \frac{\ell(Q)^{\varepsilon}}{|Q|}\left(\int_{Q} \frac{\left\|b_{Q}-b(y)\right\|}{|x-y|^{n+\varepsilon}} d y\right) d x \\
& \leq \frac{\ell(Q)^{\varepsilon}}{|Q|} \int_{Q}\left\|b_{Q}-b(y)\right\|\left(\int_{(2 Q)^{c}} \frac{d x}{|x-y|^{n+\varepsilon}}\right) d y \\
& \leq \frac{\ell(Q)^{\varepsilon}}{|Q|} \int_{Q}\left\|b_{Q}-b(y)\right\|\left(\int_{|x|>\ell(Q)} \frac{d x}{|x|^{n+\varepsilon}}\right) d y \leq C\|b\|_{B M O} .
\end{aligned}
$$

Since $\left\|\int_{Q} b(y) a(y) d y\right\| \leq \frac{1}{|Q|} \int_{Q}\left\|b(y)-b_{Q}\right\| d y$ we can estimate

$$
\begin{aligned}
\sigma_{3,2}(x) & \leq \chi_{(2 Q)^{c}}(x)\left\|k\left(x, x_{Q}\right)\right\|\|b\|_{B M O} \\
& \leq\|b\|_{B M O} \chi_{(2 Q)^{c}}(x) \gamma\left(\left|x-x_{Q}\right|^{n}\right) .
\end{aligned}
$$

Therefore one gets

$$
\begin{aligned}
\left|\left\{x: \sigma_{3,2}(x)>\lambda\right\}\right| & \leq\left|\left\{x \in(2 Q)^{c}: \gamma\left(\left|x-x_{Q}\right|^{n}\right)>\|b\|_{B M O}^{-1} \lambda\right\}\right| \\
& =\left|\left\{x \in(2 Q)^{c}:\left|x-x_{Q}\right|<\left[\gamma^{-1}\left(\|b\|_{B M O}^{-1} \lambda\right)\right]^{1 / n}\right\}\right| .
\end{aligned}
$$

This gives the estimate $\left|\left\{x: \sigma_{3,2}(x)>\lambda\right\}\right| \leq \psi^{-1}\left(\|b\|_{B M O}^{-1} \lambda\right)=\alpha(\lambda)$. The proof is then easily concluded.

\section{References}

[B] S. Bloom, A commutator theorem and weighted BMO, Trans. Amer. Math. Soc. 292(1) (1985), 103-122.

[CRW] R. R. Coifman, R. Rochberg, and G. Weiss, Factorization theorems for Hardy spaces in several variables, Ann. of Math. (2) 103(3) (1976), 611-635. 
[CP] D. Cruz-Uribe and C. PÉrez, Two-weight, weak-type norm inequalities for fractional integrals, Calderón-Zygmund operators and commutators, Indiana Univ. Math. J. 49(2) (2000), 697-721.

[FS] C. Fefferman and E. M. Stein, $H^{p}$ spaces of several variables, Acta Math. 129(3-4) (1972), 137-193.

[GR] J. García-Cuerva And J. L. Rubio DE Francia, "Weighted norm inequalities and related topics", North-Holland Mathematics Studies 116, Notas de Matemática [Mathematical Notes] 104, North-Holland Publishing Co., Amsterdam, 1985.

[HST] E. Harboure, C. Segovia, and J. L. Torrea, Boundedness of commutators of fractional and singular integrals for the extreme values of $p$, Illinois J. Math. 41(4) (1997), 676-700.

[P1] C. PÉREZ, Endpoint estimates for commutators of singular integral operators, J. Funct. Anal. 128(1) (1995), 163-185.

[P2] C. PÉREZ, Sharp estimates for commutators of singular integrals via iterations of the Hardy-Littlewood maximal function, J. Fourier Anal. Appl. 3(6) (1997), 743-756.

[PP] C. PÉREz AND G. Pradolini, Sharp weighted endpoint estimates for commutators of singular integrals, Michigan Math. J. 49(1) (2001), 23-37.

[PT1] C. PÉrez and R. Trujillo-GonzÁlez, Sharp weighted estimates for multilinear commutators, J. London Math. Soc. (2) 65(3) (2002), 672-692.

[PT2] C. PÉRez And R. Trujillo-GonzÁlez, Sharp weighted estimates for vector-valued singular integral operators and commutators, Tohoku Math. J. (2) 55(1) (2003), 109-129.

[RRT] J. L. Rubio de Francia, F. J. Ruiz, and J. L. Torrea, Calderón-Zygmund theory for operator-valued kernels, Adv. in Math. 62(1) (1986), 7-48.

[ST1] C. Segovia And J. L. Torrea, Vector-valued commutators and applications, Indiana Univ. Math. J. 38(4) (1989), 959-971.

[ST2] C. Segovia And J. L. Torrea, A note on the commutator of the Hilbert transform, Rev. Un. Mat. Argentina 35 (1989), 259-264 (1991).

[ST3] C. Segovia And J. L. TorreA, Weighted inequalities for commutators of fractional and singular integrals, Conference on Mathematical Analysis (El Escorial, 1989), Publ. Mat. 35(1) (1991), 209-235.

[ST4] C. Segovia And J. L. Torrea, Commutators of LittlewoodPaley sums, Ark. Mat. 31(1) (1993), 117-136. 
[ST5] C. Segovia And J. L. Torrea, Higher order commutators for vector-valued Calderón-Zygmund operators, Trans. Amer. Math. Soc. 336(2) (1993), 537-556.

[SW] E. M. SteIn AND G. WeIss, "Introduction to Fourier analysis on Euclidean spaces", Princeton Mathematical Series 32, Princeton University Press, Princeton, N.J., 1971.

Departament de Matemàtiques

Universitat de València

Burjassot 46100, València

Spain

E-mail address: oscar.blasco@uv.es

Rebut el 22 de gener de 2008. 